\title{
APPLICATION OF RAPID PROTOTYPING TECHNOLOGY IN THE MANUFACTURING OF TURBINE BLADE WITH SMALL DIAMETER HOLES
}

\author{
Mariusz Deja ${ }^{1}$ \\ Michał Dobrzyński ${ }^{1}$ \\ Paweł Flaszyński² \\ Jacek Haras ${ }^{1}$ \\ Dawid Zieliński ${ }^{1}$ \\ 1 Gdańsk University of Technology, Faculty of Mechanical Engineering, \\ Department of Manufacturing and Production Engineering, Poland \\ 2 Institute of Fluid Flow Machinery, Polish Academy of Sciences, Poland
}

\begin{abstract}
The article presents the possibilities++ of using Rapid Prototyping (RP) technology in the manufacturing of turbine blades with small diameter holes. The object under investigation was gas turbine blade with small diameter cooling holes and holes for generating longitudinal vortices. A turbine blade model was produced by means of Direct Metal Laser Sintering (DMLS) technology and subsequently validated in terms of detection and accuracy of the obtained holes. The application of the computer tomography and digital radiography technique resulted in obtaining a series of cross-sections of the turbine blade model with a series of holes. Particular attention was pointed out at the investigation of the locations of micro-holes with a diameter of $0.3 \mathrm{~mm}$. It turned out that it was impossible to make such small holes by the RP method. In the following part the results of the study on the possibilities of making the micro-holes using electrical discharge method have been presented. In addition, proposition of further works such as the development of the considerations and issues discussed in this article, has been offered.
\end{abstract}

Keywords: turbine blade, small diameter holes, Rapid Prototyping/Rapid Manufacturing, Direct Metal Laser Sintering (DMLS) technology

\section{INTRODUCTION}

The Rapid Prototyping/Rapid Manufacturing technologies consisted of sets of methods and additive techniques making product manufacturing and prototyping possible, are ones of the most dynamically developing methods for parts forming and manufacturing. Unlike the conventional deductive machining methods, the rapid prototyping and manufacturing technologies allow to obtain an element on the basis of its spatial numerical model worked out by means of a CAD system. The conventional deductive machining technologies have limitations especially in the machining of higher-order surfaces and small-diameter holes, in particular of different angular positions.

Superimposing subsequent material layers on each other during rapid prototyping process result in reaching complete product whose shape and form follow its $3 \mathrm{D}$ geometry designed by a CAD system. One of the main merits of application of the Rapid Prototyping/Rapid Manufacturing methods is the forming of prototypes and physical models of products and their use in every stage of product developing process - beginning from concept up to repair. Such approach makes it possible not only to limit costs resulting from incorrectly manufactured product 
series but also just in the first stage of product development, i.e. its design stage, to conduct crucial investigations and tests, that allows to elaborate an optimum designed product.

Application of additive techniques allows to produce a very broad spectrum of parts connected e.g. with overhaul or failure of devices installed on ships or offshore steel structures. An advantage of application of the technology is possibility of producing metal elements of complex forms at limited working space required for a device used in rapid prototyping. Devices used in rapid prototyping do not require any special instrumentation but is able to replace several machine tools used in deductive technologies. Rapid Prototyping/Rapid Manufacturing method is of special importance in manufacturing complex geometry products, especially those having higher-order surfaces characteristic a.o. for blades of high-pressure stages of gas turbines intended for ship propulsion or Tesla turbines $[1,3,4,7]$. Apart from the complex geometry they have holes and ducts for cooling medium flow, whose manufacturing with the use of deductive methods is often impossible.

There are today many methods for rapid prototyping and manufacturing.

The techniques vary both in respect of form and type of processed material, way of building physical model as well as source of energy generated for additive process $[8,9]$. One of the most dynamically developing additive methods is the direct metal powder laser sintering technology DMLS (Direct Metal Laser Sintering) which was patented by EOS (Electro Optical Systems), a German firm. The method consists in the local melting of material powder and next joining superimposed subsequent layers of material on each other into a uniform solid due to solidifying process. The main advantage of application of the method is the possible manufacturing of products and moulds of complex shapes which, due to their geometrical complexity, are difficult or impossible to be produced by means of traditional deductive or casting methods [2,12]. Another of them is EBM (Electron Beam Melting) method which resembles the DMLS process but, instead of laser sintering, electron beam is used in it for metal powder bonding. The EBM technology is utilized for manufacturing the industrial rotors of complex geometry and thin walls. [6]. The SLM technology (Selective Laser Melting) makes it possible to produce precise parts at maintaining uniform internal structure of material, its density and strength $[10,11]$.

\section{OBJECT AND METHODS OF INVESTIGATIONS}

The object of investigations was a turbine blade model designed in Institute of Fluid Flow Machinery, Polish Academy of Sciences (IMP PAN), Gdańsk, which was then manufactured by Intelligent Technologies Unit (ZTI), the Institute of Fundamental Technical Problems, Polish Academy of Sciences (IPPT PAN), Warsaw. Blades of high pressure stages of gas turbines used in ship and aircraft drives as well as power systems operate in high temperature conditions. One of the essential structural elements of the this way loaded blades are properly designed holes ensuring cooling medium flow and effective blade material separation from hot gases. In grid of blades working medium flow is accelerated up to supersonic velocities that results in generating shock wave on blade suction side, whose interaction with boundary layer may initiate formation of separation zones and flow recirculation in boundary layer area. The interaction between shock wave and boundary layer in presence of cooling film and strong pressure gradients forces to search for flow control methods in order to improve flow system's life and rise its efficiency. Such target of investigations was formulated in IMP PAN where a design of gas turbine blade fitted with cooling holes and holes intended for generating longitudinal vortices, was prepared [1]. In view of geometrical complexity an attempt was made to manufacture the profile with cooling medium holes and ducts by applying the DMLS technique.

The turbine blade model was produced by means of DMLS technique with the use of EOS Maraging Steel MS1 in the form of powder, i.e. a hardenable martensitic tool steel of content complying with EU standard 1.2709 [13]. Production of the turbine blade model was executed by using EOSINT M280 machine for which the following process parameters were selected:

- thickness of single layer of laid material $-40 \mu \mathrm{m}$,

- total number of model material layers - 3834,

- type and power of used laser - a fibrous ytterbium laser of $400 \mathrm{~W}$ effective power,

- length of laser beam - 1060-1100 nm.

Additional tests were conducted to check if it is possible to made a series of micro-holes of $0.3 \mathrm{~mm}$ in diameter by using a material deductive method - spark drilling because it turned out that production of the designed holes by applying $\mathrm{RP} / \mathrm{RM}$ technology was unsuccessful. To this end, a highspeed HGH EDM Drill 528 DZ spark drilling machine which ensures high manufacturing accuracy for small-diameter holes, was used. The drilling process was carried out with the following parameters:

- wire diameter $-0.3 \mathrm{~mm}$,

- extreme electric current value - $5 \mathrm{~A}$,

- condenser capacity ( single pulse power) - 4 C,

- spark pause ( pause between subsequent pulses) - $3 \mathrm{~s}$.

Fig. 1 presents an image of the designed turbine blade model with indicated arrangement of holes being the object of the investigations in question. The analyzed geometry took into account holes of $3 \mathrm{~mm}, 2.5 \mathrm{~mm}, 2 \mathrm{~mm}, 1.35 \mathrm{~mm}$, $1 \mathrm{~mm}$ in diameter and micro-holes of $0.3 \mathrm{~mm}$ in diameter. Worth paying attention to their different angular positions which resulted from constructional purposes, i.e. in order to take into account operational factors of turbine blades. The manufacturing of a series of micro-holes of $0.3 \mathrm{~mm}$ diameter was especially important for possible application of DMLS technology in the production field. 


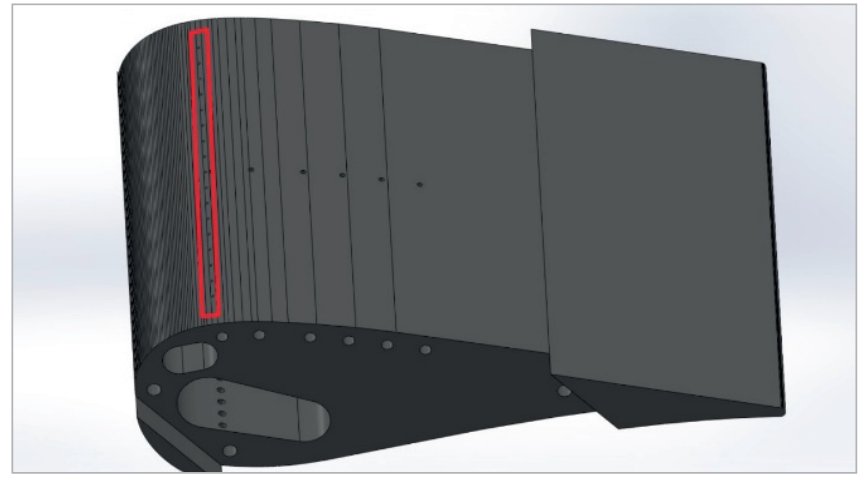

Fig. 1.3D image of turbine blade

\section{RESULTS OF INVESTIGATIONS}

The application of computer tomography as well as digital radiography made it possible to perform series of measurements for turbine blade model. The achieved results allowed to make analysis of geometry of the research turbine blade model and detection of holes and micro-holes manufactured by DMLS method.

The computer tomography imaging was carried out by means of Nikon XT H 450 tomograph in Smart Solutions firm [5]. An example image of longitudinal cross-section of the turbine blade model is presented in Fig. 2.

The conducted tests allowed to carry out measurements of two rows of holes of $1.35 \mathrm{~mm}$ diameter. Additionally, based on the achieved image, distances between neighbouring holes were also determined. In order to determine possibility of manufacturing the micro-holes by means of DMLS method the computer tomography tests were also performed in transverse direction. The obtained results are shown in Fig. 3. The presented image shows the holes produced in one of the turbine blade walls. On the basis of the carried out measurements it may be stated that at the assumed parameters of sintering process the micro-holes of the assumed diameter equal to $0.3 \mathrm{~mm}$ and angular position were not gained.

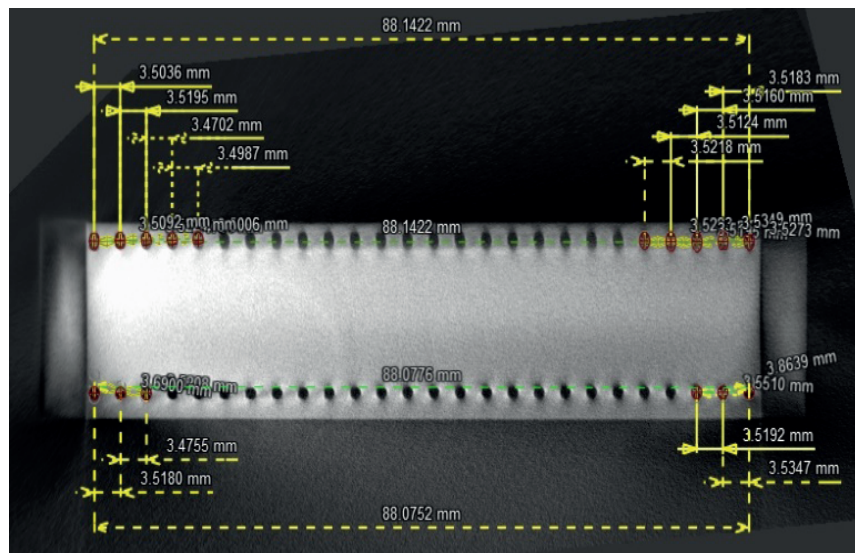

Fig. 2. Measurement results for holes of 1,35 $\mathrm{mm}$ diameter. Image of turbine blade cross-section in longitudinal direction
Measurements with the use of digital radiography were performed to make detail analysis of manufacturing holes and micro-holes. The test was carried out with the use of Andreks $300 \mathrm{~kW}$ radiograph in the Bonding Engineering Unit laboratory, Mechanical Faculty, Gdańsk University of Technology.

Fig. 4 shows a radiogram where the holes obtained in the turbine blade made by using additive method may be observed.

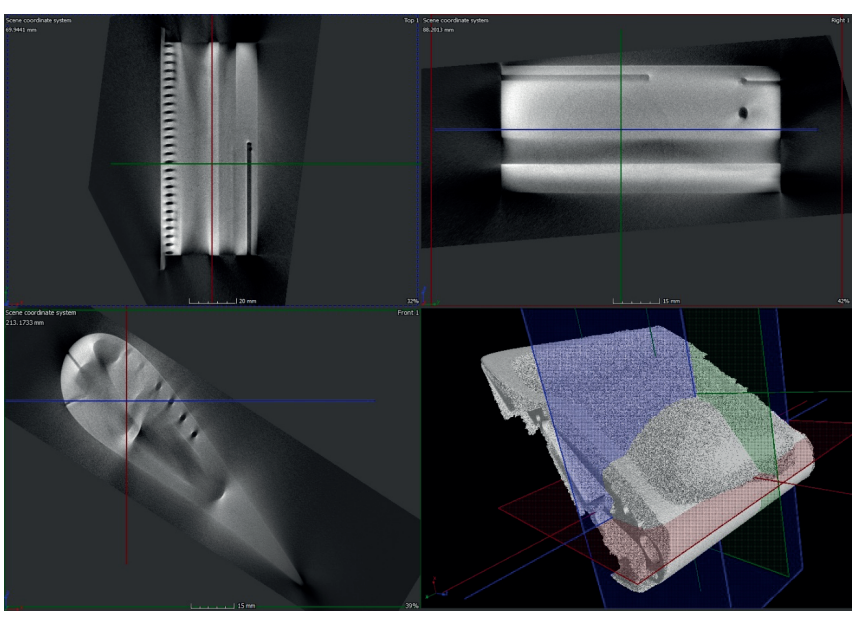

Fig. 3. Exemplary images of cross-section of turbine blade model

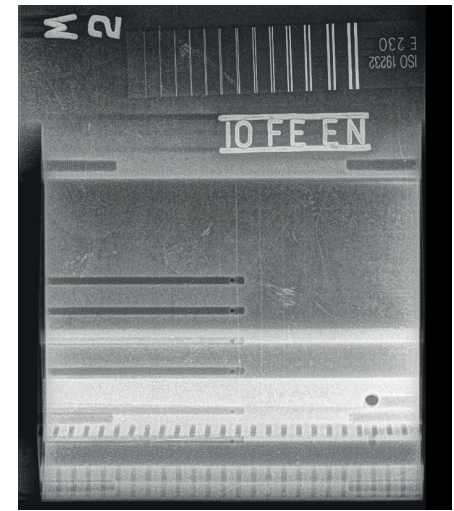

Fig. 4. Radiogram with visible holes obtained in turbine blade made by using additive method

The conducted digital radiography tests made it possible to detect and measure the holes in the diameter range from $1 \mathrm{~mm}$ to $3 \mathrm{~mm}$. Tab. 1 contains a set of measurement results for the holes with taking into account their number and diameters.

Tab. 1. Set of measurement results obtained by means of digital radiography

\begin{tabular}{|c|c|c|}
\hline No. & Number of holes & Hole diameter $[\mathrm{mm}]$ \\
\hline 1 & 2 & 2.5 \\
\hline 2 & 6 & 2 \\
\hline 3 & 4 & 2.5 \\
\hline 4 & 6 & 1 \\
\hline 5 & 1 & 3 \\
\hline 6 & 26 & 1.35 \\
\hline 7 & 26 & 1.35 \\
\hline
\end{tabular}


The results obtained from the conducted digital radiography tests confirm the results achieved from computer tomography. In the turbine blade cross-sections no micro-holes of $0.3 \mathrm{~mm}$ diameter were found. To examine spots of their forming more precisely a micro-magnifying glass of $1 \times 30$ magnification was used. Exemplary spots of forming micro-holes on blade surface are presented in Fig. 5 a) and 5 b).

a)

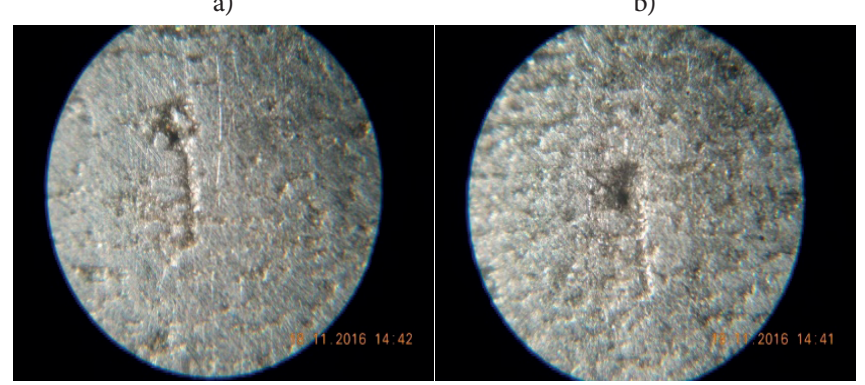

Fig. 5. Spots of forming the micro-holes of $0.3 \mathrm{~mm}$ diameter on blade surface: a) a nucleus of micro-hole, b) a tear-off on material surface

Analyzing the obtained measurement results one can observe only nuclei of micro-holes, that indicates a lack in complete metal powder melting. Additionally, material continuity faults in the form of tear-offs are also observed. On the basis of the performed investigations it was stated that the application of DMLS technology at the set values of additive process parameters did not result in producing micro-holes of $0.3 \mathrm{~mm}$ diameter.

In the subsequent stage of the investigations micro-holes of $0.3 \mathrm{~mm}$ diameter were produced by means of spark drilling (electric discharge) technique whose possible application and precision in manufacturing micro-holes was next validated.

The results of measurements with the use of digital radiography indicate that the formed micro-holes are of $0.4 \mathrm{~mm}$ diameter, i.e. somewhat greater than that assumed. Therefore the use of spark drilling technology did admittedly allow to obtain micro-holes but the dimensional accuracy requirement was not fulfilled.

The achieved image of turbine blade cross-section with marked series of micro-holes produced by spark drilling technique is presented in Fig. 6.

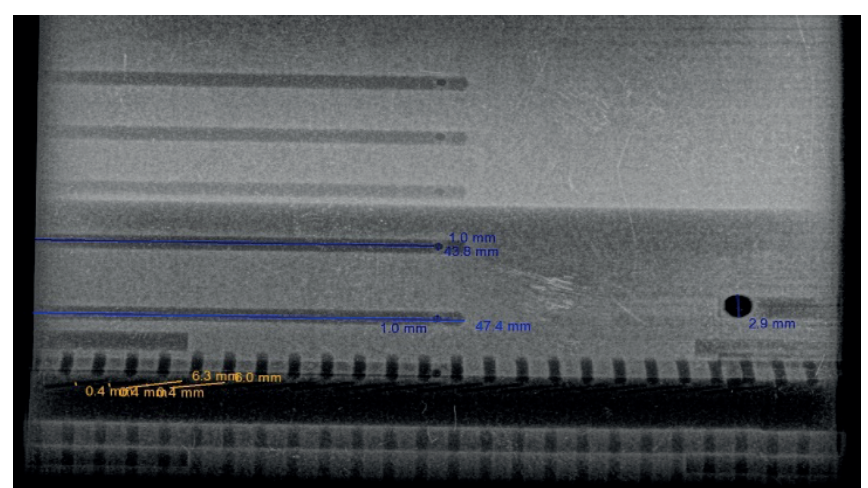

Fig. 6. Micro-holes of $0.3 \mathrm{~mm}$ diameter produced by means of spark drilling technique

\section{CONCLUSIONS}

Rapid Prototyping/Rapid Manufacturing technologies constitute, in view of their dynamic development, a very important area of today-used methods for products forming and manufacturing. The techniques in question make it possible to work out prototypes and physical models of objects of any shape on the basis of their 3D geometry. These technologies constitute an important element in product development cycle as they allow to do a reliable assessment in every stage of its development.

The application of the selective laser melting method (DMLS) made it possible to produce a real model of gas turbine blade with cooling holes and holes for longitudinal vortices generating. The DMLS technology allowed to produce a series of holes of $3 \mathrm{~mm}, 2.5 \mathrm{~mm}, 2 \mathrm{~mm}, 1.35 \mathrm{~mm}$ and $1 \mathrm{~mm}$ in diameter under different angles directly during additive process. A lack in complete metal melting did not allow to produce a series of micro-holes of $0.3 \mathrm{~mm}$ diameter, revealing this way limitations in application of the selective laser melting technology to making holes of a diameter smaller than $1 \mathrm{~mm}$. The additionally performed spark drilling operation did not allow to produce micro-holes just of $0.3 \mathrm{~mm}$ diameter but only of $0.4 \mathrm{~mm}$ diameter. Application of an electrode of smaller diameter would allow probably to reach holes of the required dimensional accuracy. The searching for a relation between electrode diameter and dimensional and shape accuracy of produced holes will be a subject of further investigations.

The carrying out of analysis of turbine blade model geometry is a basis for decision making on further investigations dealing with utilization of rapid prototyping methods and deductive methods in the area of micro-holes manufacturing.

The next step is to prepare a test specimen especially designed for this purpose.

An appropriate analysis of the test model geometry will allow to determine possibilities and limitations for application of Rapid Prototyping/Rapid Manufacturing technologies to direct manufacturing micro-holes of a diameter less than $1 \mathrm{~mm}$.

\section{BIBLIOGRAPHY}

1. Flaszynski P., Doerffer P., Piotrowicz M.: Effect of Jet Vortex Generators on Shock Wave Induced Separation on Gas Turbine Profile. Proceedings of the $13^{\text {th }}$ International Symposium on Experimental Computational Aerothermodynamics of Internal Flows, 7-11 May 2017, Okinawa, Japan.

2. Gebhard J.: Generative Fertigungsverfahren, Additive Manufacturing und 3D Drucken für Prototyping-ToolingProduktion; Prototyping-Tooling-Produktion, Carl Hanser Verlag, München 2013.

3. Iftikhar A., Khan M., Alam K., Imran Jaffery S. H., Ali L., Ayaz Y. and Khan A.: Turbine blade manufacturing through rapid tooling (RT) process and its quality inspection. 
Materials and Manufacturing Processes, Vol. 28, Issue 5, (2013), pp. 534-538.

4. Lampart P., Kosowski K., Piwowarski M. and Jędrzejewski Ł.: Design analysis of Tesla micro-turbine operating on a low-boiling medium. Polish Maritime Research, Special issue 2009/S1; pp. 28-33.

5. Nikon XT H series, X-ray technology and CT for industrial applications, Nikon Metrology/ Vision Beyond Precision, 2017.

6. Tan X., Kok Y., Tor S. B. and Chua, C. K.: Application of Electron Beam Melting (EBM) in Additive Manufacturing of an Impeller. Proceedings of the $1^{\text {st }}$ International Conference on Progress in Additive Manufacturing (ProAM 2014), 2014, pp. 327-332.

7. Vaezi M., Safaeian D. and Chua C.K.: Gas turbine blade manufacturing by use of epoxy resin tooling and silicone rubber moulding techniques. Rapid Prototyping Journal, Vol. 17 Issue: 2, 2011, pp.107-115.

8. Vayre B., Vignat F. and Villeneuve F.: Designing for Additive Manufacturing. 45th CIRP Conference on Manufacturing Systems, Procedia CIRP 3, 2012, pp. 632-637.

9. VDI 3405:2014-12: Additive manufacturing processes, rapid manufacturing - Basics, definitions, processes.

10. Yadroitsev I. and Yadroitsava I.: Evaluation of residual stress in stainless steel 316L and Ti6Al4V samples produced by selective laser melting. Virtual and Physical Prototyping. Vol. 10, No. 2, 2015, pp. 67-76.

11. Yakout M., Elbestawi M.A. and Veldhuis S.C.: On the characterization of stainless steel 316L parts produced by selective laser melting. The International Journal of Advanced Manufacturing Technology,2017, pp 1-22.

12. https://drukarki3d.pl/technologie/dmls/

13. https://drukarki3d.pl/materialy/dmls/eos-maragingsteel-msl/

\section{CONTACT WITH THE AUTHORS}

\author{
Mariusz Deja \\ e-mail:mariusz.deja@pg.gda.pl \\ Michał Dobrzyński \\ Jacek Haras \\ Dawid Zieliński
}

Gdańsk University of Technology

Faculty of Mechanical Engineering

Department of Manufacturing and Production Engineering

11/12 Narutowicza St. 80-233 Gdańsk

Poland

Paweł Flaszyński

Institute of Fluid Flow Machinery

Polish Academy of Sciences

14 Fiszera St.

80-231 Gdańsk

POLAND 